\title{
EFFICIENCY OF ARCHITECTURAL VOIDS ON NATURAL VENTILATION IN MULTI-STOREY BUILDINGS: CFD ANALYSIS
}

\author{
Ahmad Sabri El-Sayed', Ahmad Ahmad Fikri', Abbas Mohamad El-Zaafarani", \\ Khaled Ibrahim Ahmad \\ 1. Consultant Engineer for Public Buildings, MSc in Architecture, Cairo University. \\ 2. Professor of Environmental Design and Planning, Department of Architecture, \\ Faculty of Engineering, Cairo University. \\ 3. Professor of Environmental Design and Planning and former Dean of the Faculty of \\ Urban Planning, Cairo University. \\ 4. Professor, Department of Mechanical Engineering, King Abdul-Aziz University, \\ Jeddah. \\ Email: a_sabri@yahoo.com
}

\begin{abstract}
Improving the performance of natural ventilation in multi-storey buildings is important for living in terms of health and thermal comfort. One of the most important design strategies to improve natural ventilation in multi-storey buildings is by exploiting the voids inside the building. What is meant by voids here is the negative part of the built-up block such as skylights, courts or stairwells, which are usually located in the middle of the multi-storey residential building.

Therefore taking care of designing voids in buildings from the beginning is crucial in order to obtain good natural ventilation.

The experiment was conducted in this research on a four-storey hypothetically existing building. This study aims to explore the effects of voids inside the building on the efficiency of natural ventilation by incorporating two of voids forms avoiding any change in windows size or place. To assess the situation and reach the results, the experimental method was adopted and computational fluid dynamics CFD model was used. The major determinant used for assessment is the volumetric flow rate which is necessary for thermal comfort and health. This study revealed quantitatively what is known theoretically that including voids within the ventilation system and increasing their area can greatly enhance the efficiency of natural ventilation in the multi-storey building.
\end{abstract}

KEYWORDS: Natural Ventilation, Sustainability, Thermal Comfort, Building Voids, Stack Effect, Computational Fluid Dynamics CFD, Ansys Fluent, Finite Volumes 


\title{
كفاعة إدراج الفراغات المعمارية في منظومة التهوية الطبيعية للمباني متعددة الطوابق: دراسة رقمية
}

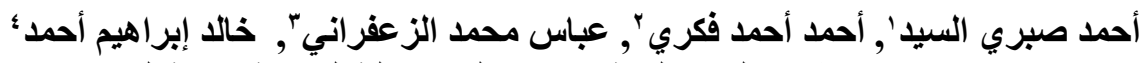

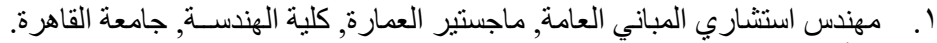

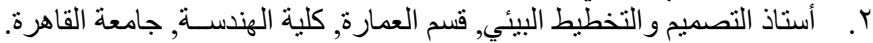

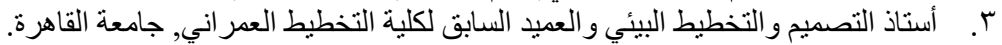

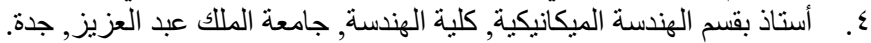 \\ البريد الإليكتروني: a sabri@yahoo.com
}

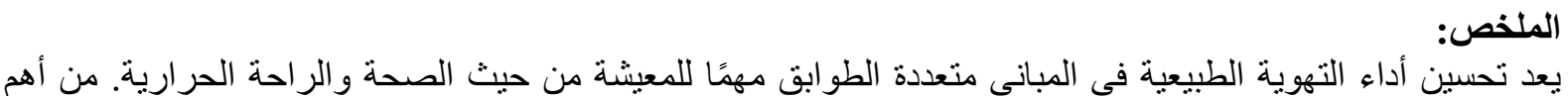

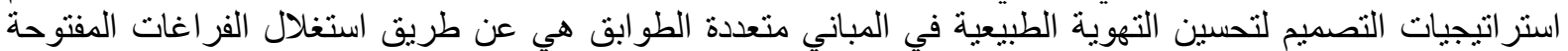

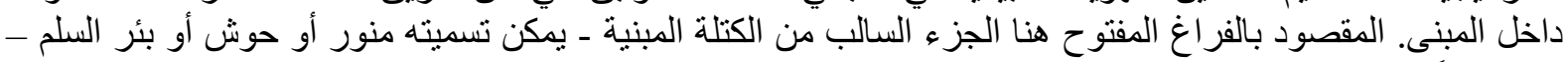

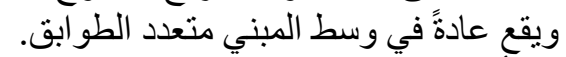

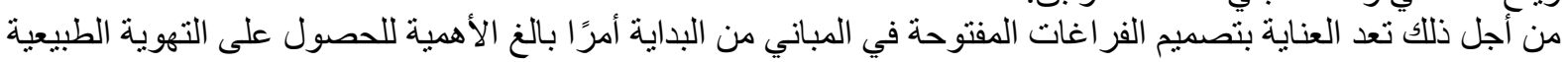
الجيدة. وقد تم اجر اء التجربة في هذا البحث على مبنى افتر اضي مكون من أربع طو ابق. تهدف هذه الدر اسة إلى استكثاف تأثير

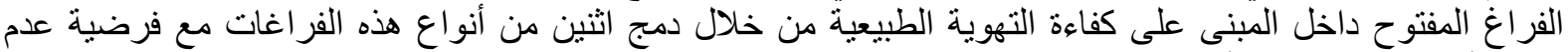

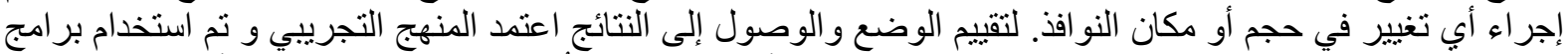

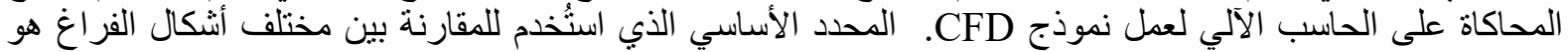

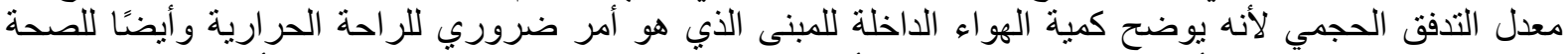

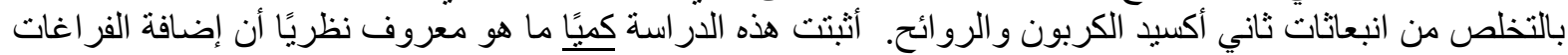
لعملية التهوية و زيادة مساحتها يمكن أن يعزز بنسب كبيرة كفاءة التهوية الطبيعية في المبنى فنئي منعدد الطوابق.

الكلمات المفتاحية: التهوية الطبيعية، الاستدامة، الراحة الحرارية، فراغات المبنى، تأثير المدخنة، ديناميكا الموائع الحسابية ، أنسيس فلوينت

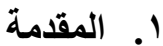

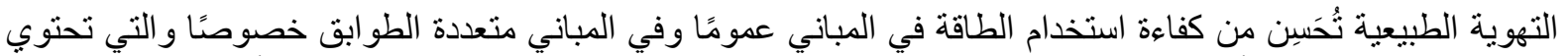

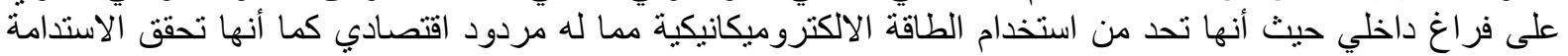

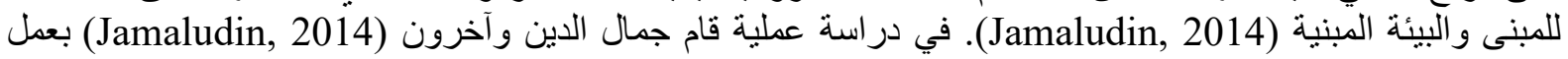

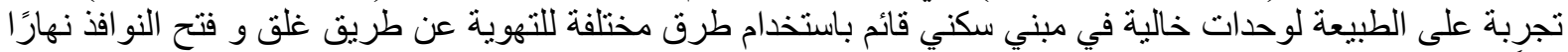

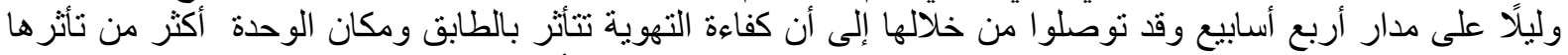

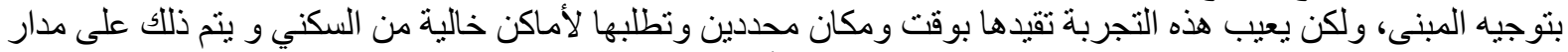

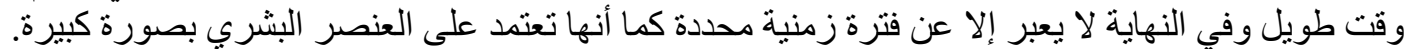

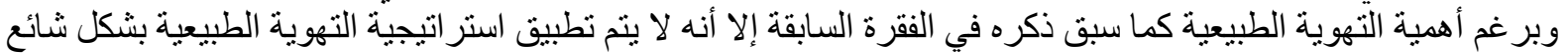

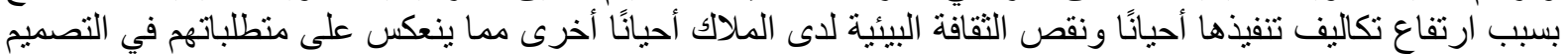

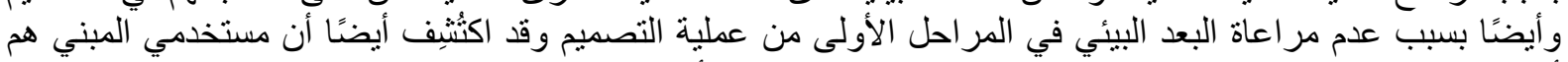

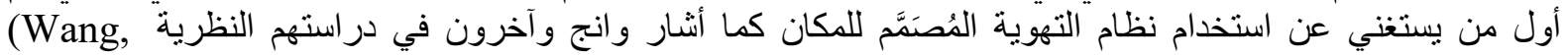

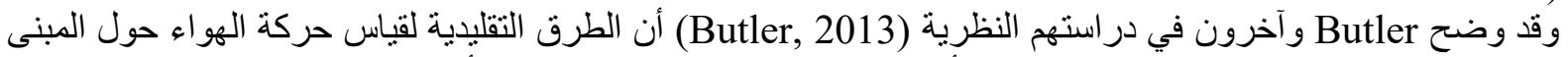

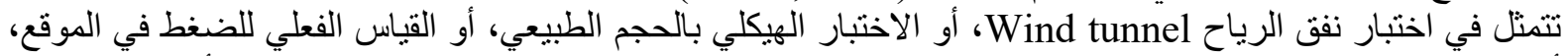

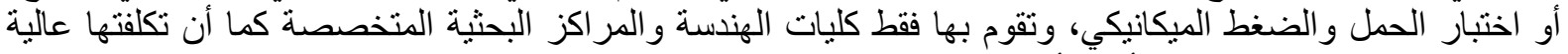

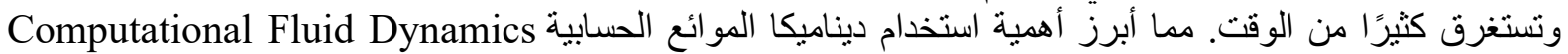

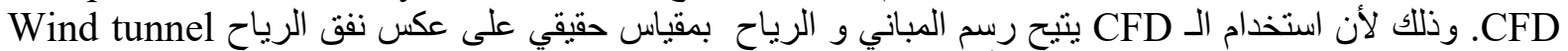

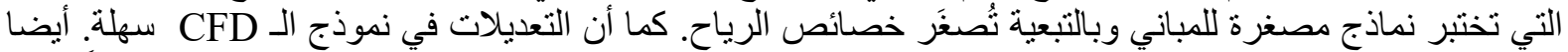

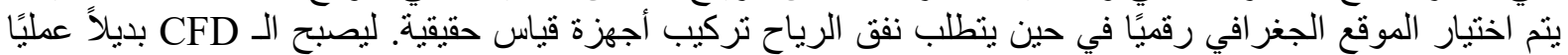

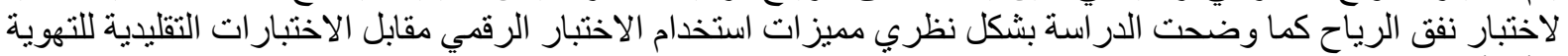

و و أنه أكثر مرونة. وقد تتاول Macquoy في در استه النظرية (Macquoy, 2014) الاستر اتيجيات العديدة الموجودة و التي تستخدم لتعزيز

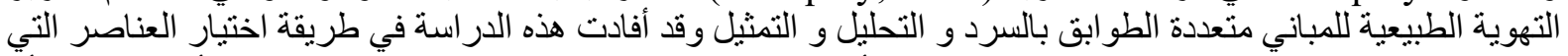
من الممكن استخدامها لتوفير التهوية الطبيعية للمبني أو استثمار ما هو موجود في المبني ألمطلوب، إلا أنه لم يختبر أياً 


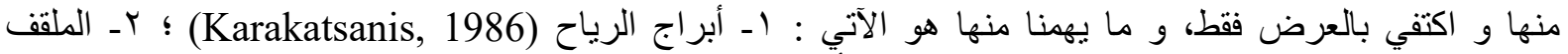

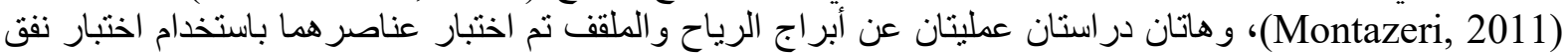

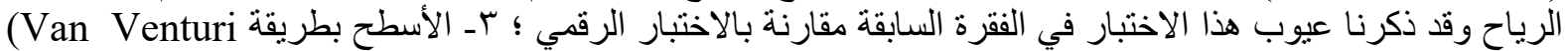

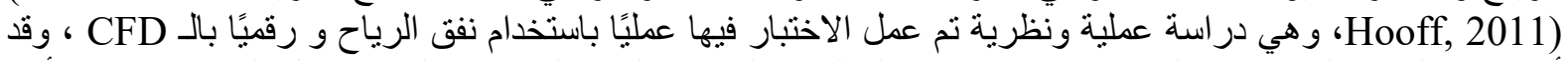

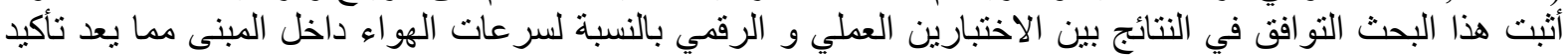

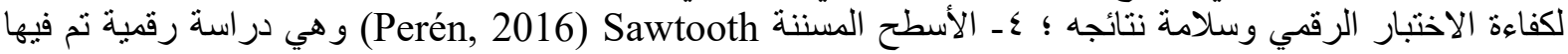

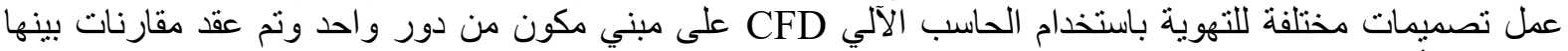

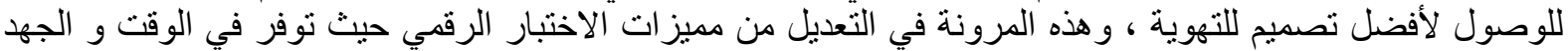

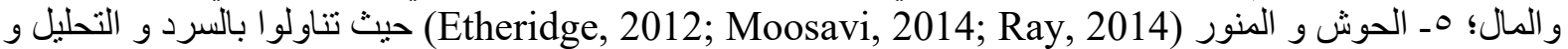

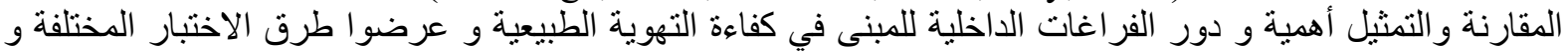

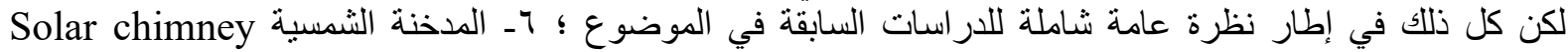
(Ahmad, 2014) وهي دراسة رقمية نم اختبار المداخن الثمسية فيها كإستر اتيجية للتهوية الطبيعية باستخدام الاختبار

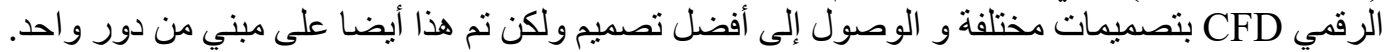

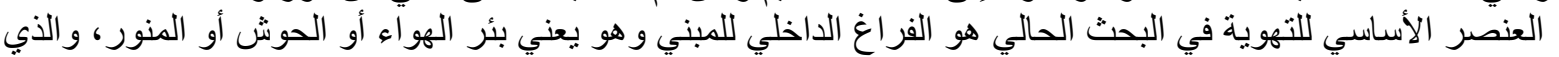

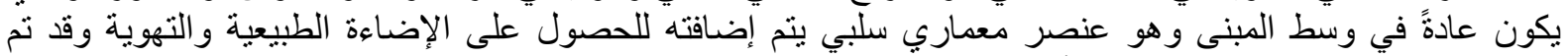

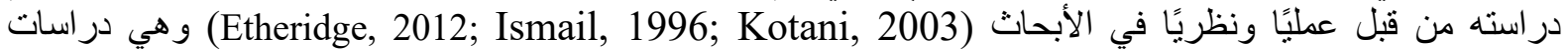

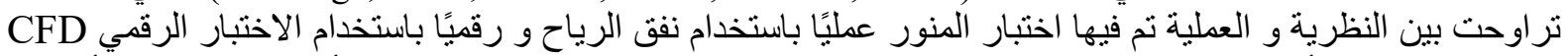

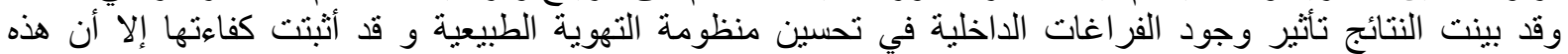

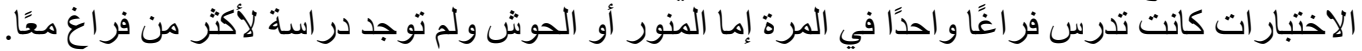

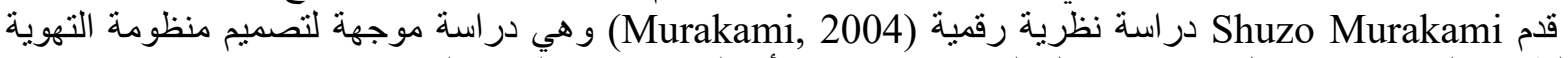

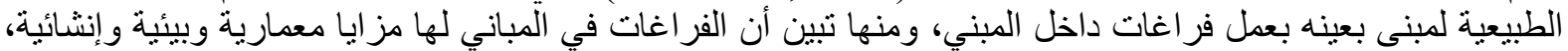

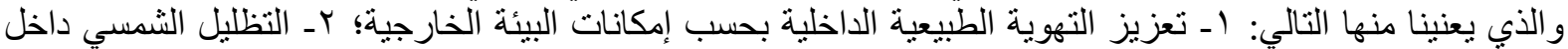

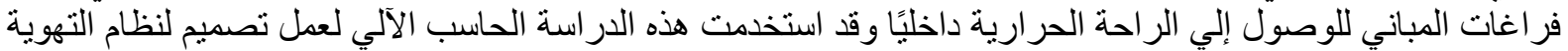

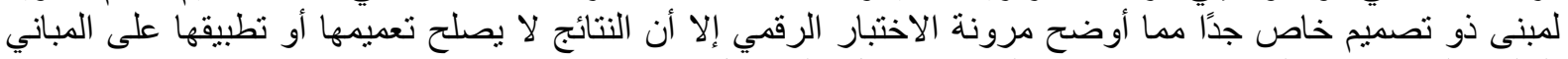

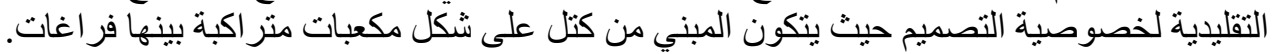

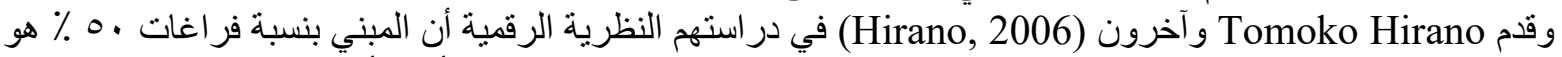

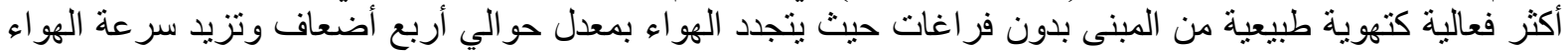

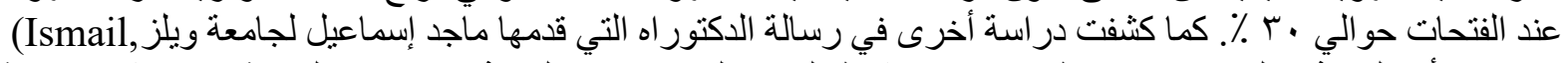
(1996 أن الفراغ يخلق فروق ضغط تتسبب في شفط الهو اء المتدفق عبر الفراغ مما يعزز التهاء التهوية. كما كثنفت نسيبة

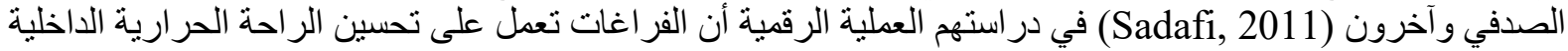

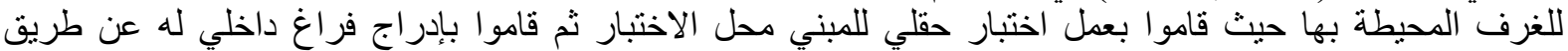

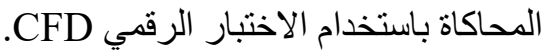

1, 1 الهُف من هذا البحث القائم

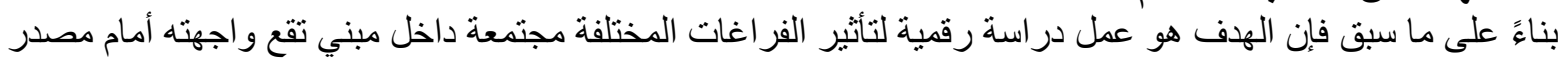

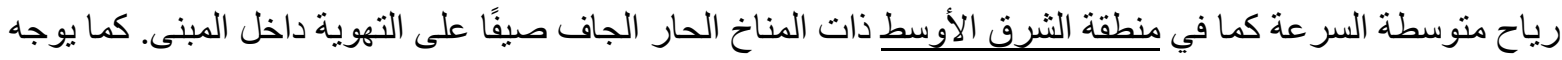

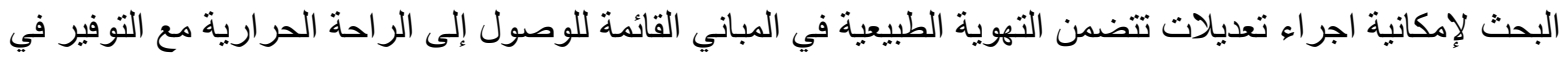

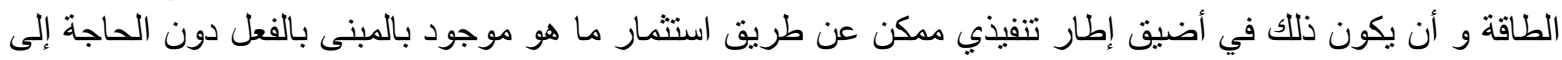

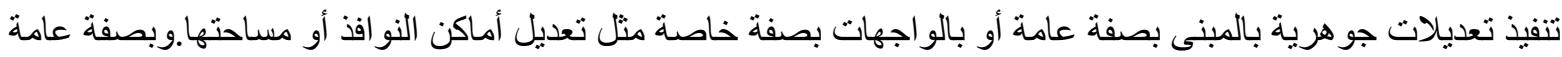

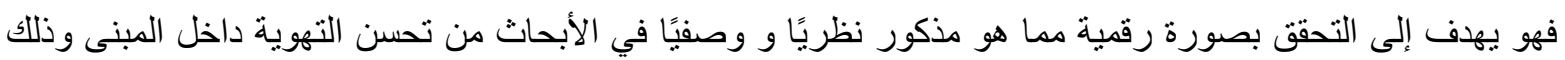

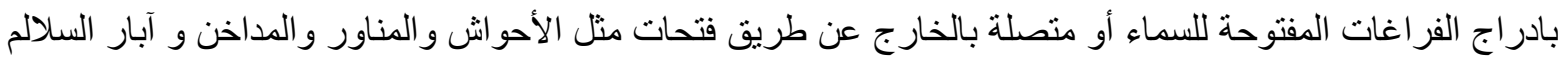

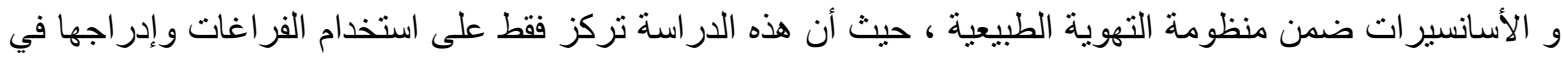

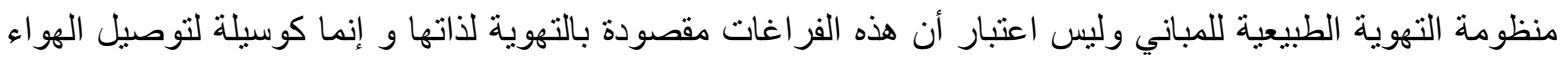

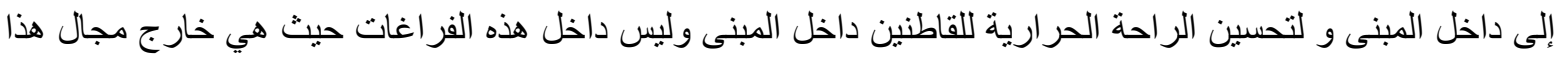


r r,l

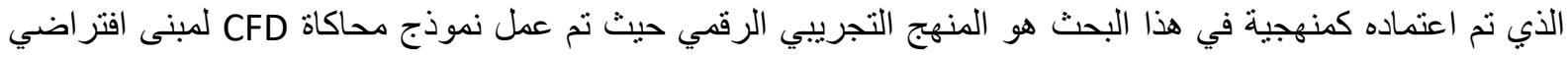

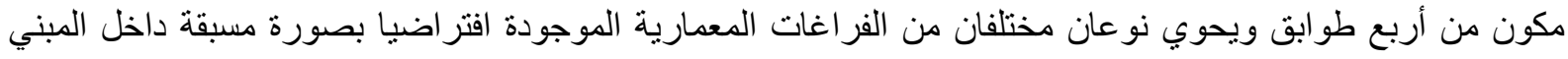

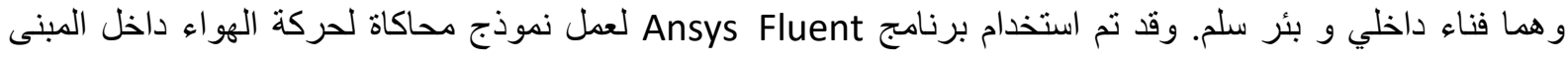

بسيناريو هات مختلفة لا تتضمن أو تتضمن إدماج الفر اغين مجتمعين أو متفرقين في منظومة التفاج التهوية الطبيعية للمبنى.

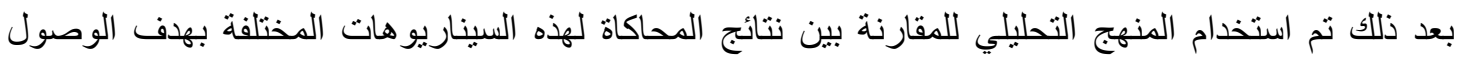

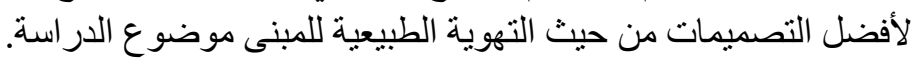

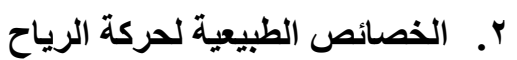

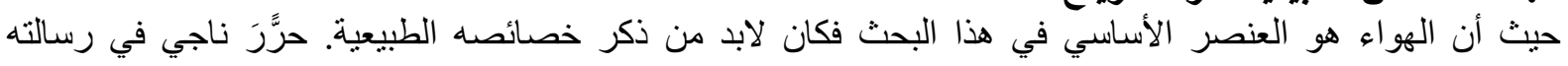

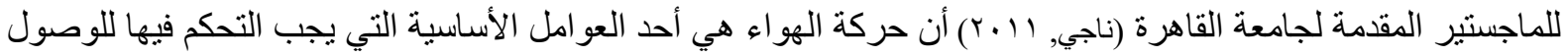

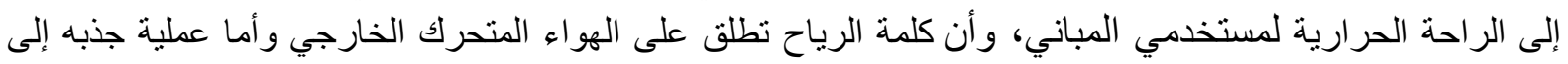

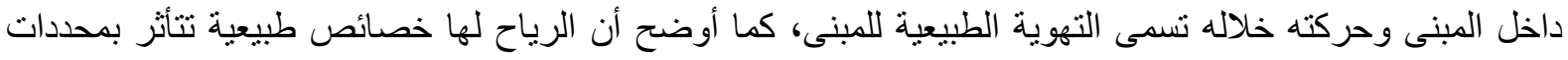

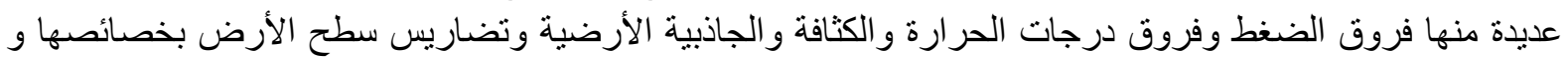

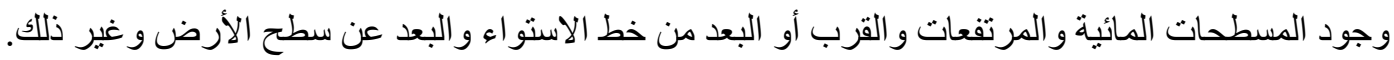

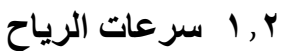

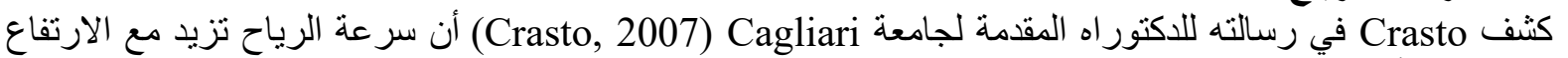

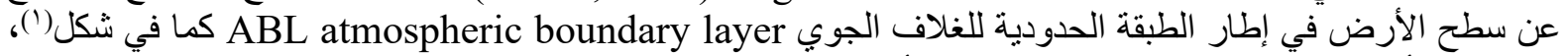

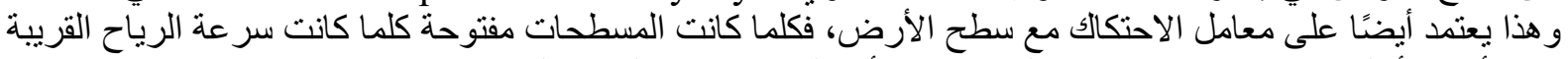

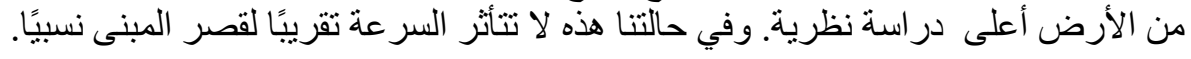

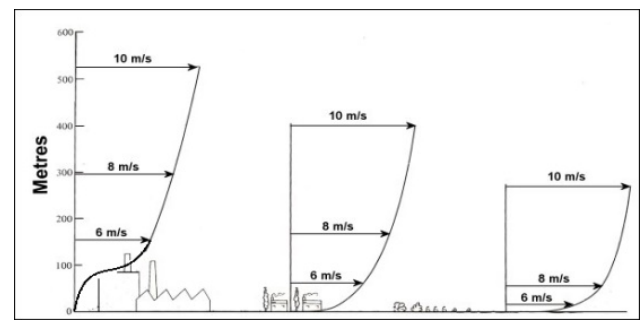

شكل ( ) منحنى سرعة الرياح

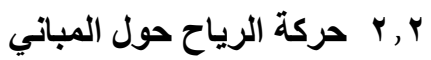

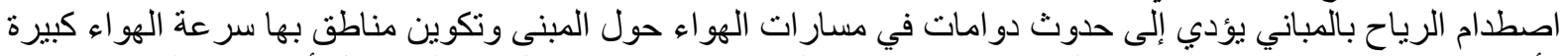

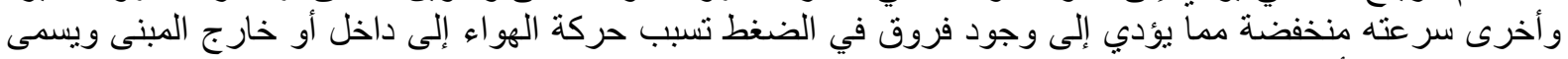

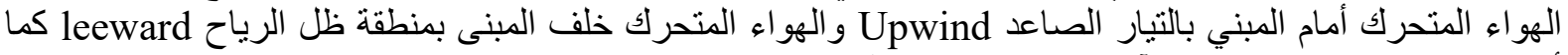
أوضح Jatupatwarangkul وآخرون في در استهم النظرية (Jatupatwarangkul, 2013).

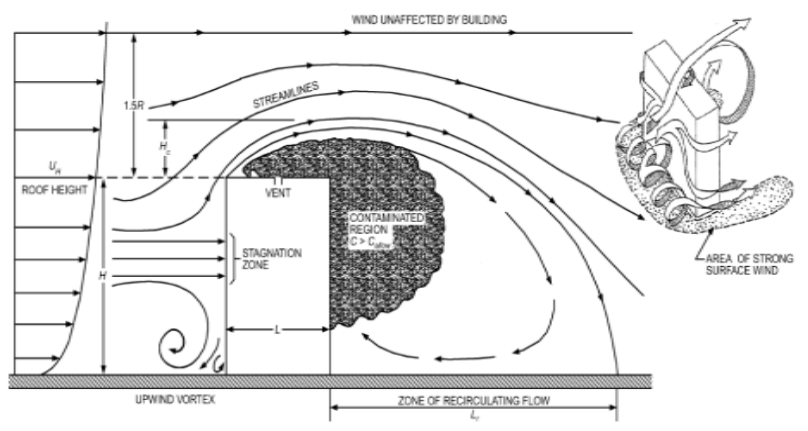

شكل (r) حركة الرياح عند اصطدامها بالمباني المرتفعة (Jatupatwarangkul, 2013) 


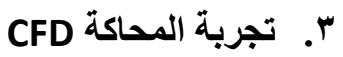

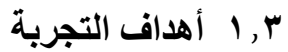

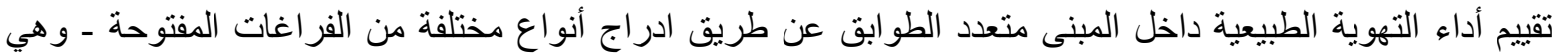

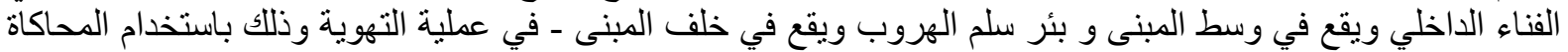

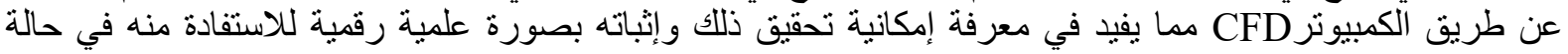
التعامل مع المباني القائمة وفي العملية التصميمية.

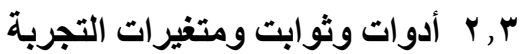

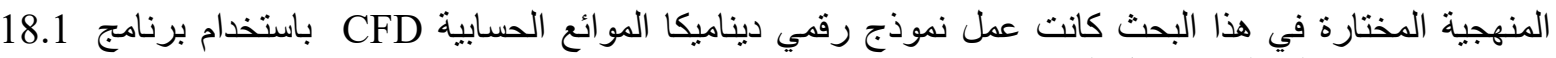
Ansys Fluent

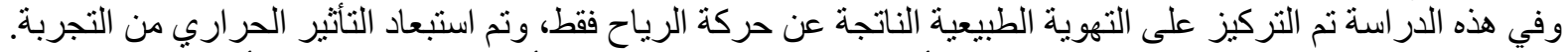

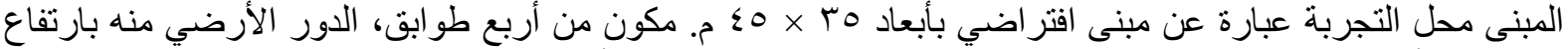

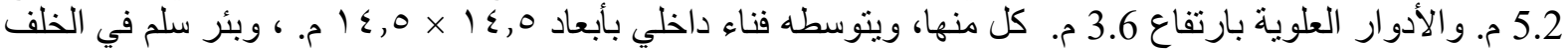

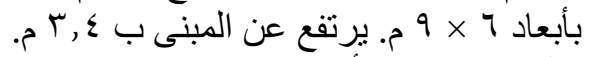

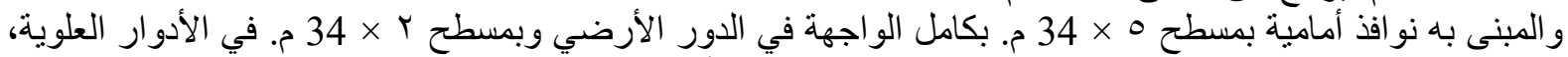

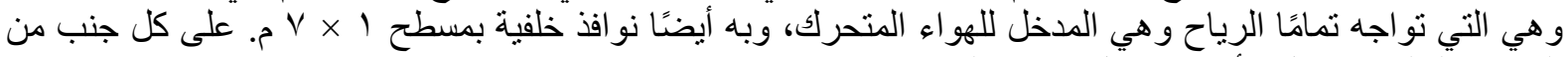

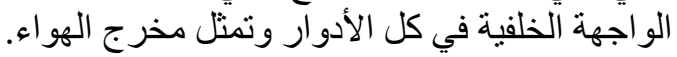

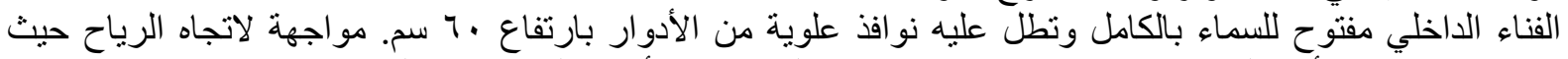

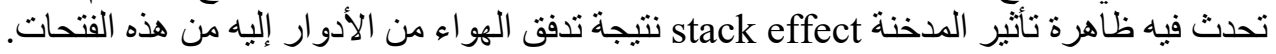

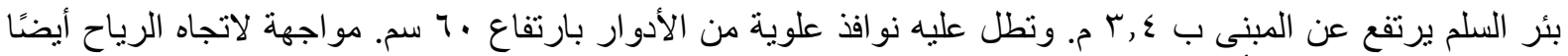

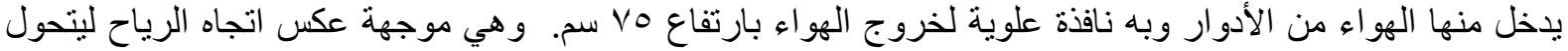

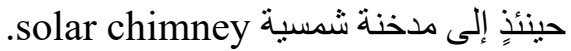

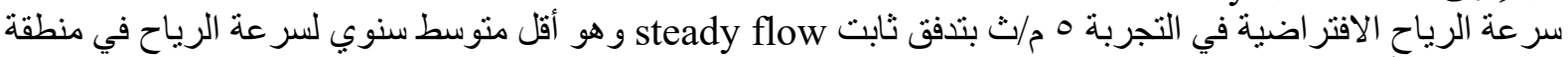

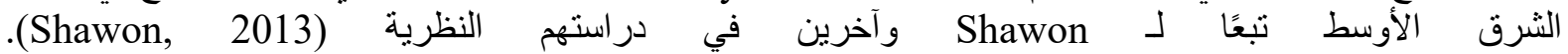
كل ما سبق ثو ابت في التجربة

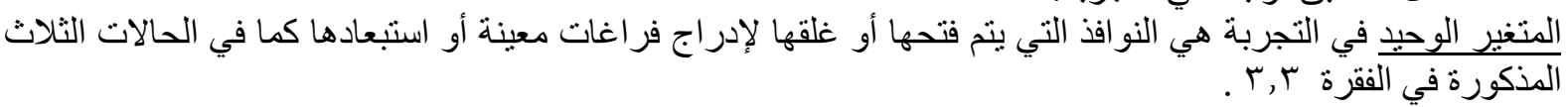

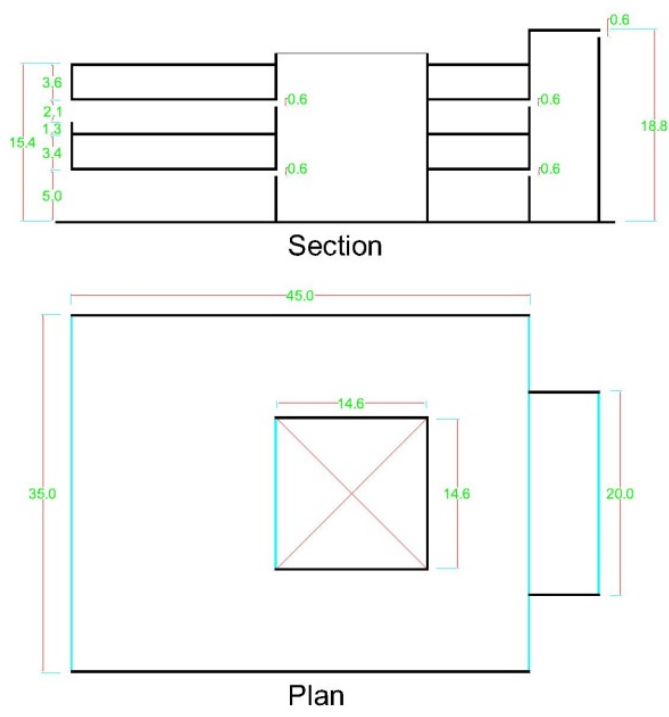

شكل (3) مسقط أفقي وقطاع للمبنى 


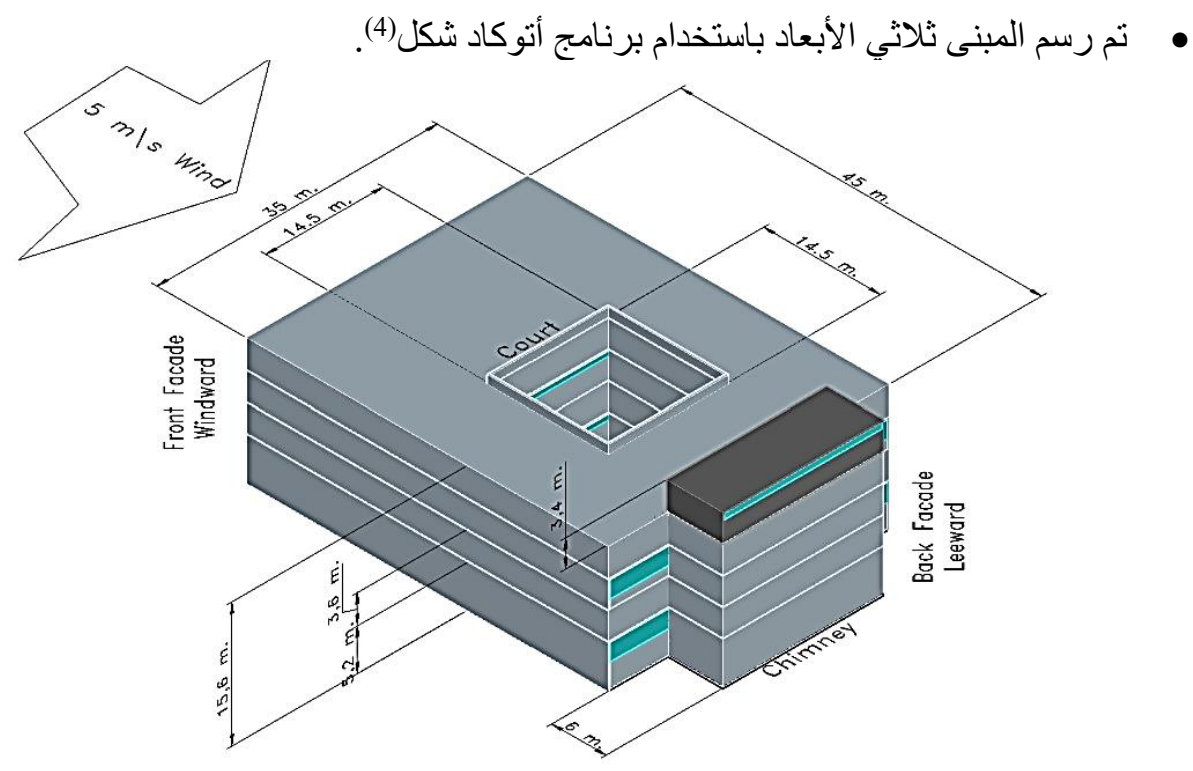

$$
\text { شكل (4) النموذج الثلاثي الأبعاد للمبنى }
$$

تم تصدير النموذج إلى برنامج 18.1 Ansys حيث يتم التعامل مع نصـف الكتلــة فقط لأنها متماثلــة ويقوم

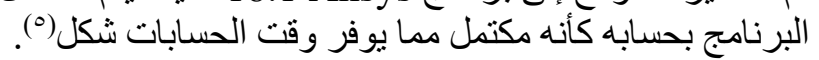

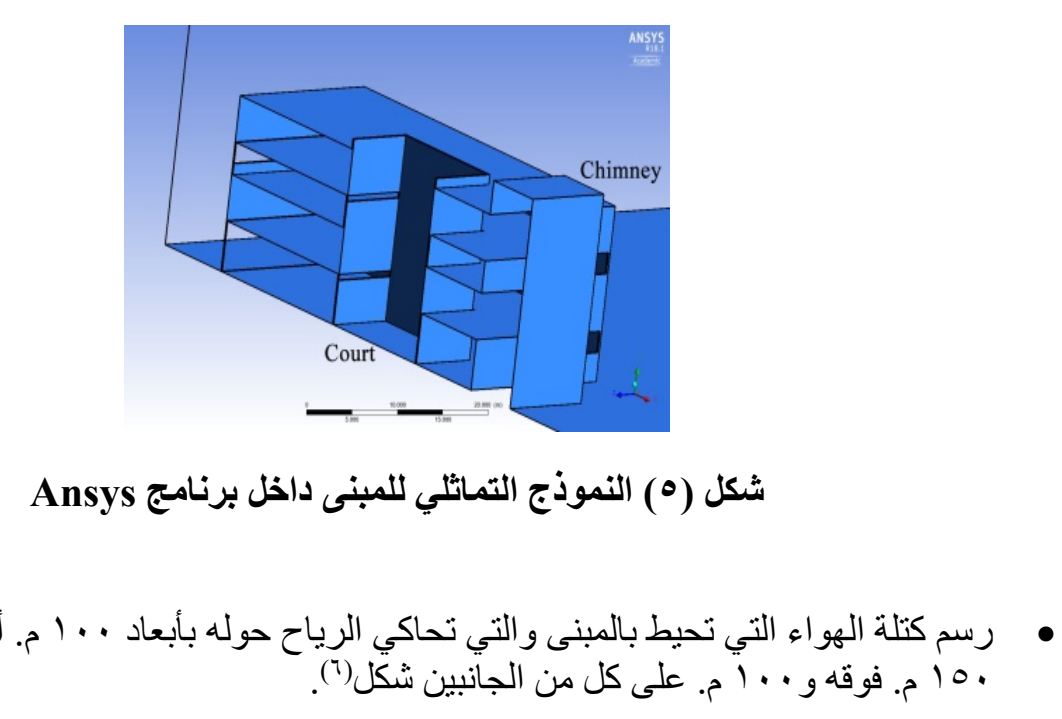

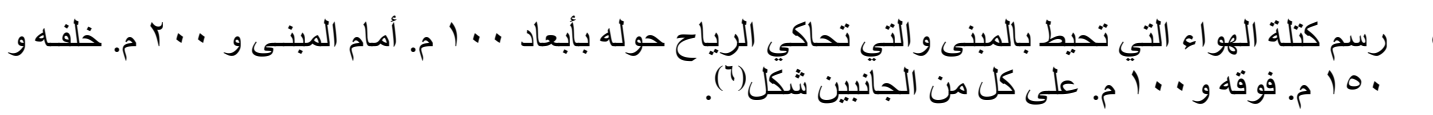




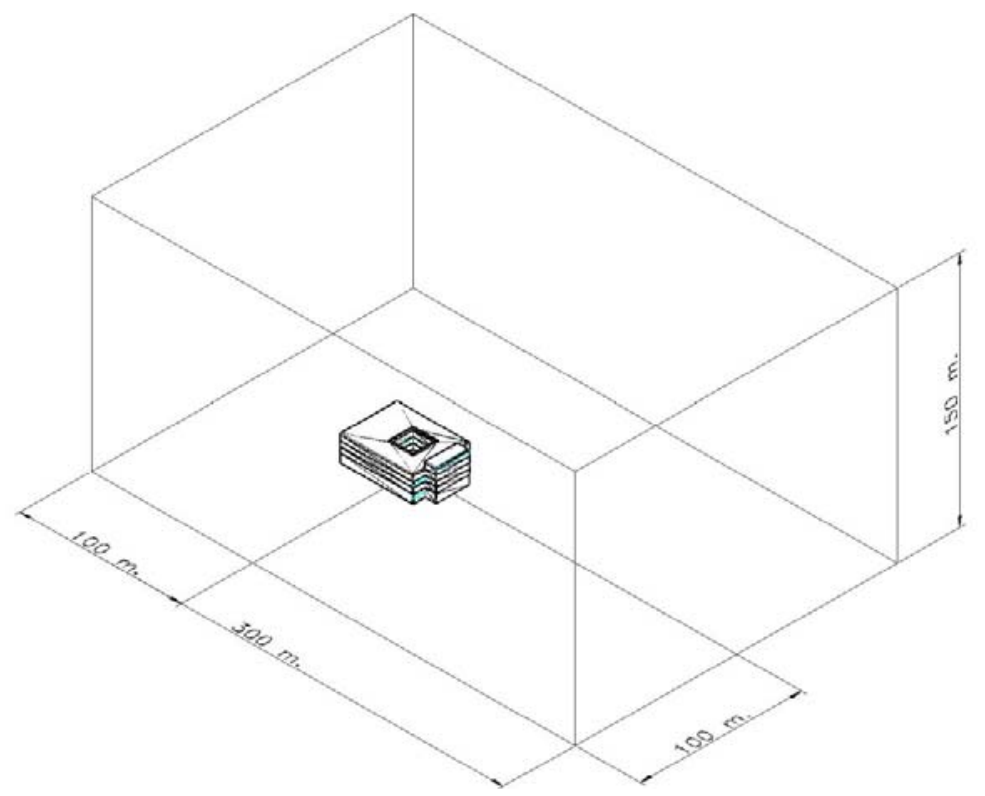

شكل (6) مجال كتلة الهواء حول المبنى (domain)

عمل رسمة شبكية meshing للنموذج ليتحول إلى حجوم محدودة finite volumes و هي مكعبات صغيرة

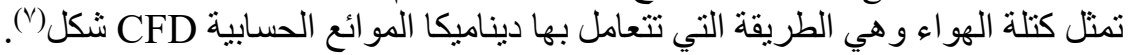

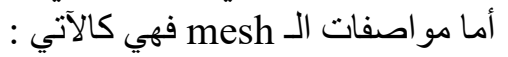

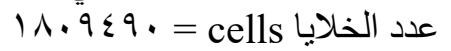
عدد الـ ع الـ عدد الـ $11 . . .09=$ nodes

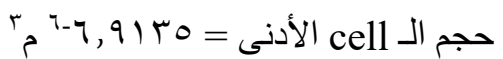

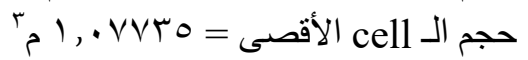
وحجم الخلية cell يتغير كلما حسب بعده عن المبنى ، فالقريب من المبنى أصغر , كما أن الحجم داخل

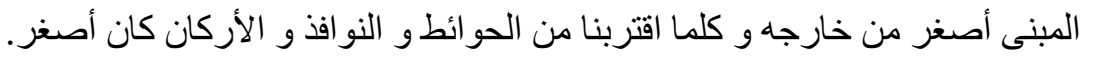

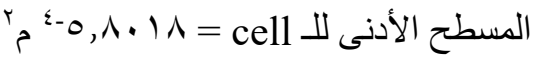

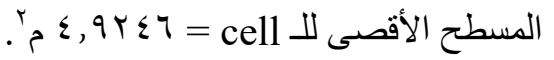

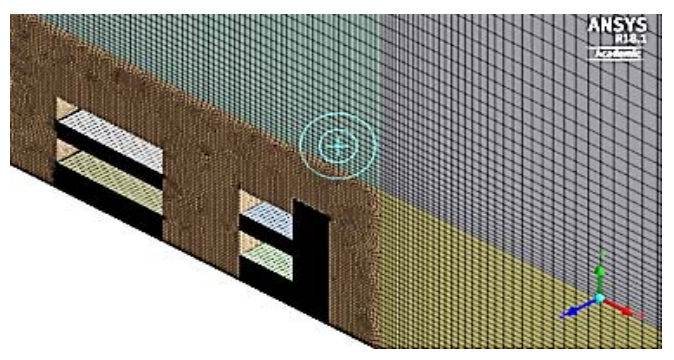

\section{Ansys شكل (7) رسمة شبكية للحجوم المحدودة للمبنى في برنامج}

الانتقال لبرنامج fluent داخل Ansys ثم اختبار الخامة لتكون هو اءو وتجهيز الموديل ليكون مزدوج الدقة و على أساس الضغطة، و على نموذج الدو امة realizable k-epsilon لأن نتائجه مستقرة. 
بعد ذللك تم وضع الثروط الحدودية boundary conditions أولًا بتحديد الحد الأمامي لمحيط الهو اء حول

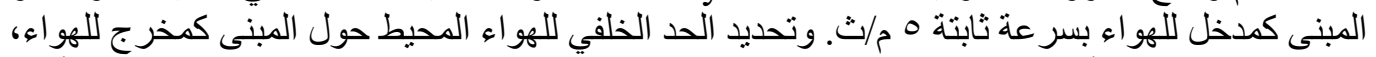

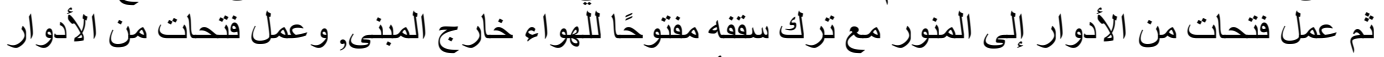

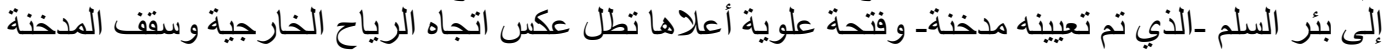
مغطى تمامًا. في خطو ات الحل داخل برنامج fluent تم اختيار الازدو اج Coupled كنظام حتى ينم حل جميع

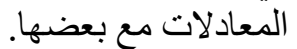

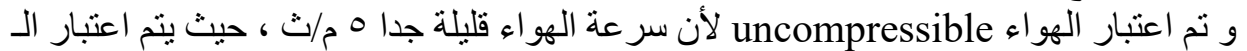
compressibility

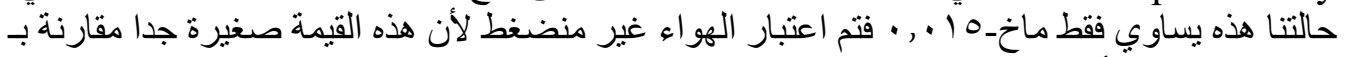

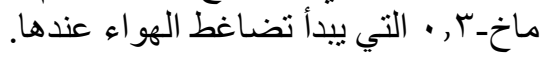
تم عمل Residual Convergence للوصول • إلى الحلى الحل كما في شكل(8).

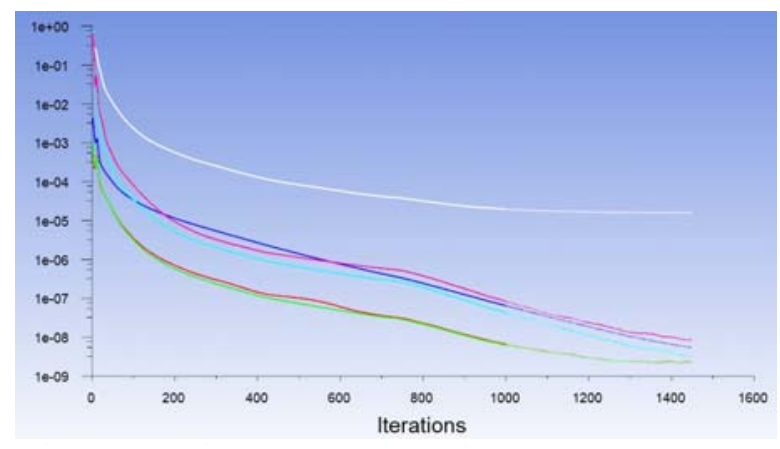

\section{شكل (8) (8) 1450 iterations, Ansys}

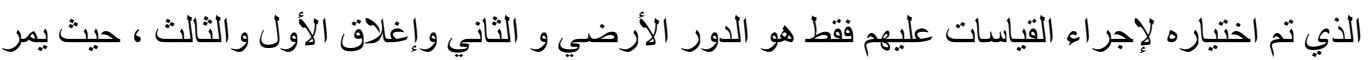

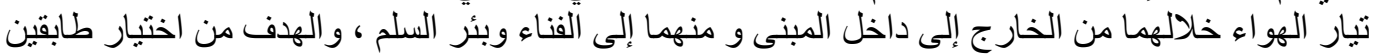

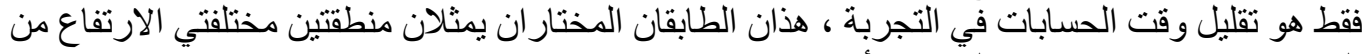
المبنى بما يحقق صورة متكاملة عن الأداء.

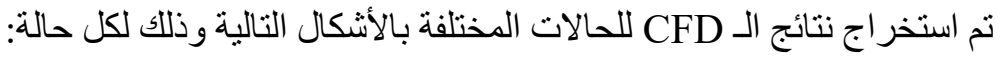

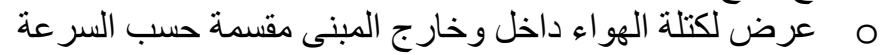
0

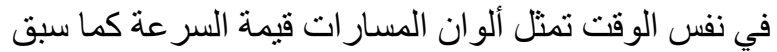
o بمر بالمنور و المدخنة o الخفية O 0

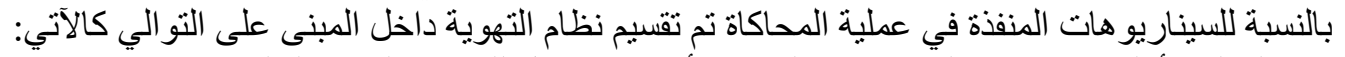

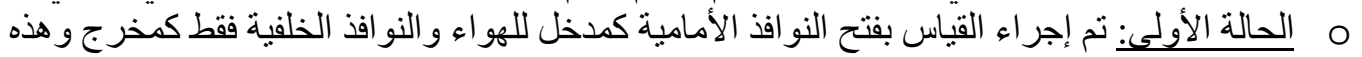
حالة التيار الهو ائي. o 0

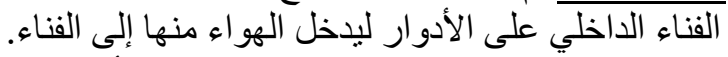

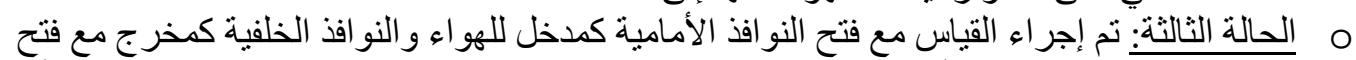

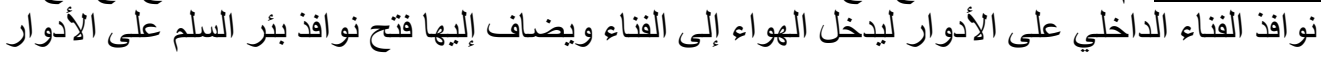

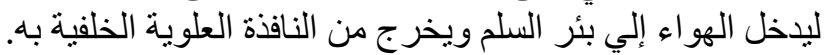

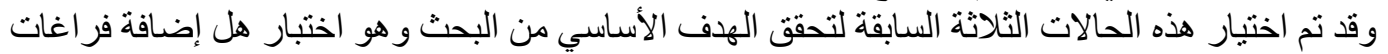

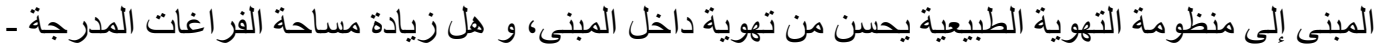




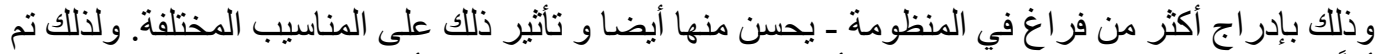

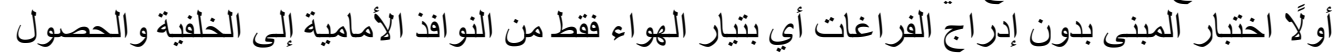

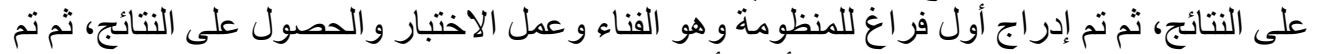

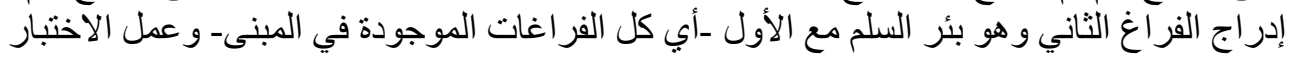

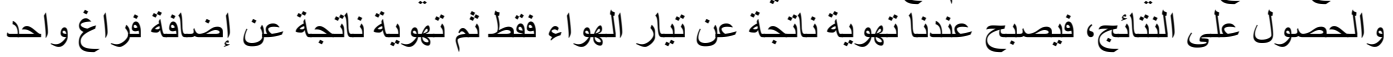

إلى المنظومة ثم تهوية ناتجة عن إضافة فر اغين إلى المنظومة، ثم ثم تم المقارنة بينهم كما سيظهر في باقي

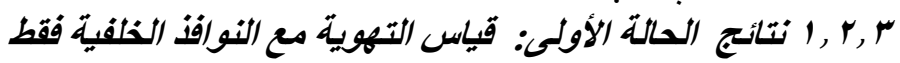

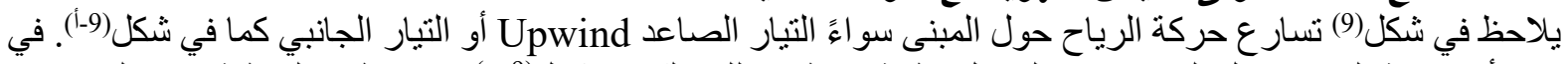

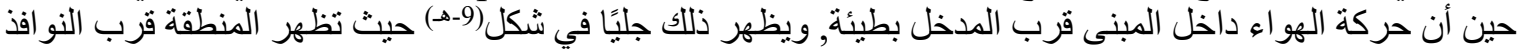

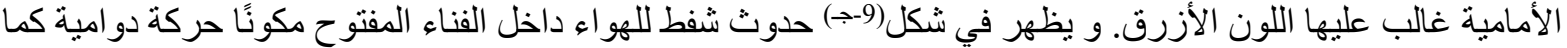

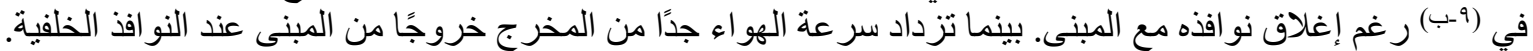

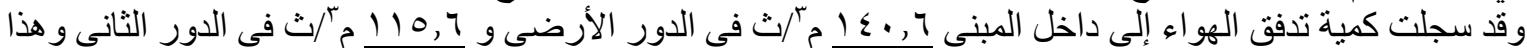
واضح في شكل(9-2).

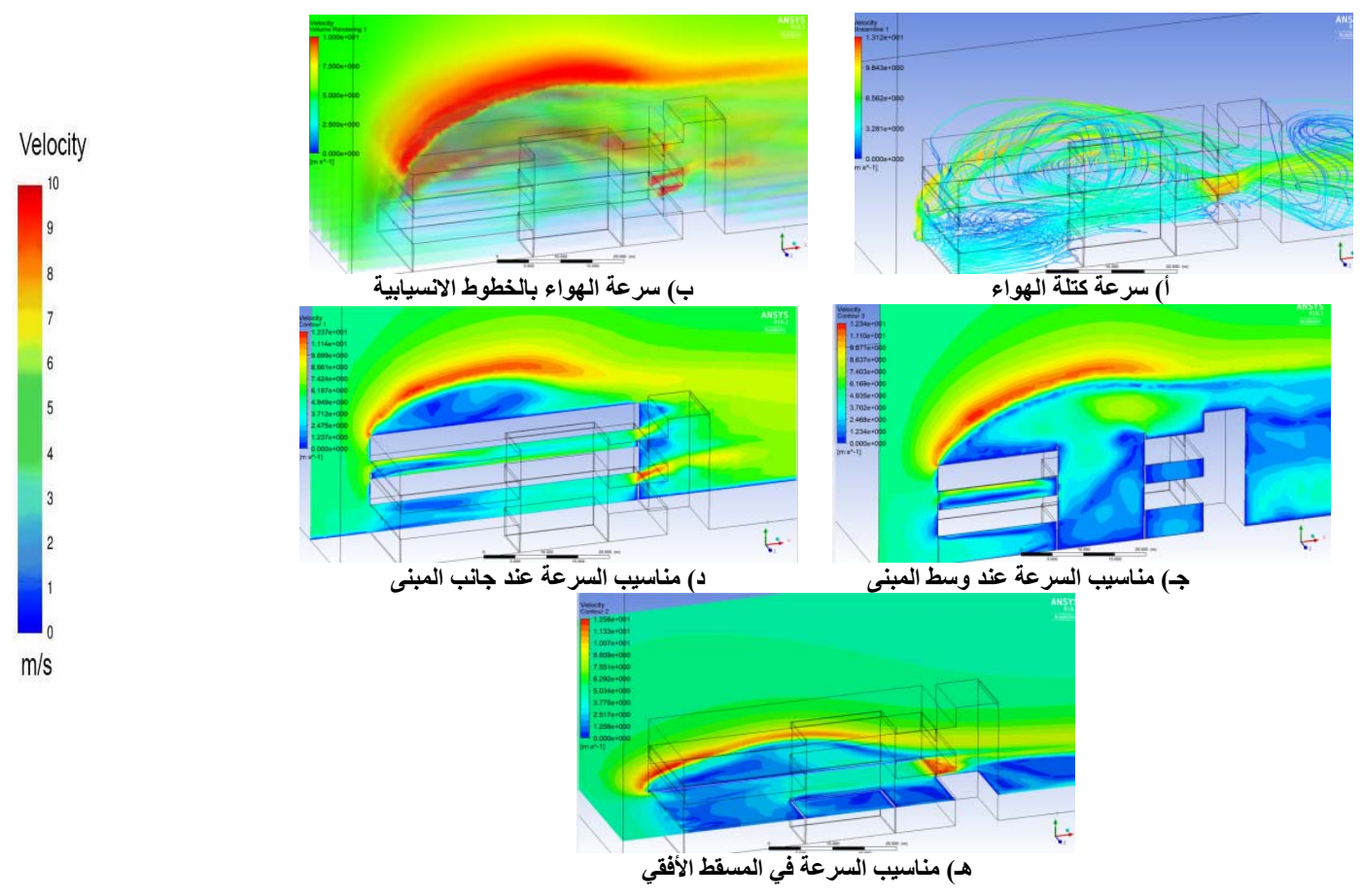

Ansys شكل (9) لقطات توضح مسار وسر عات الهواء في مقاطع مختلفة خارج وداخل المبنى للحالة الأولى, برنامج

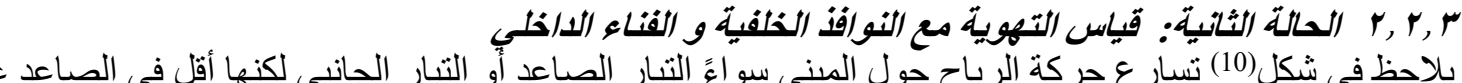

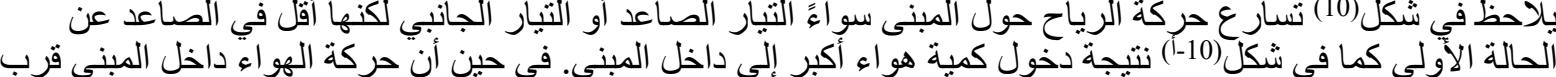

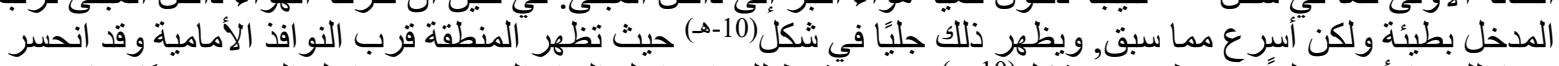

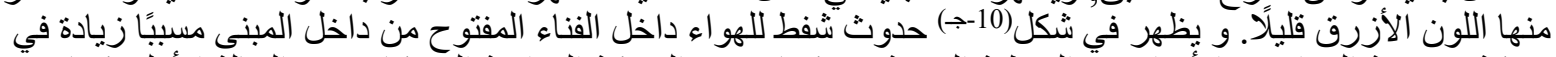

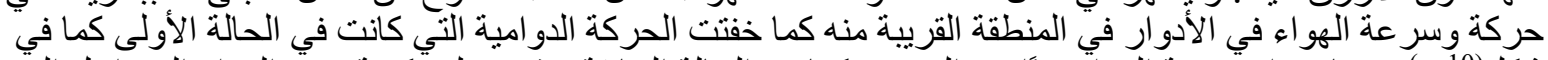

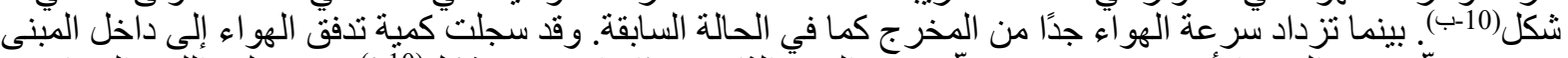

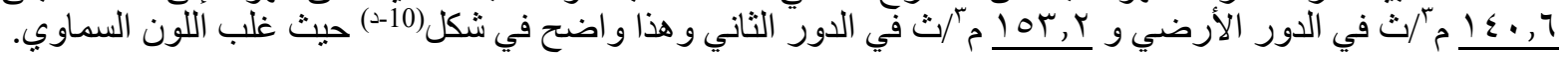




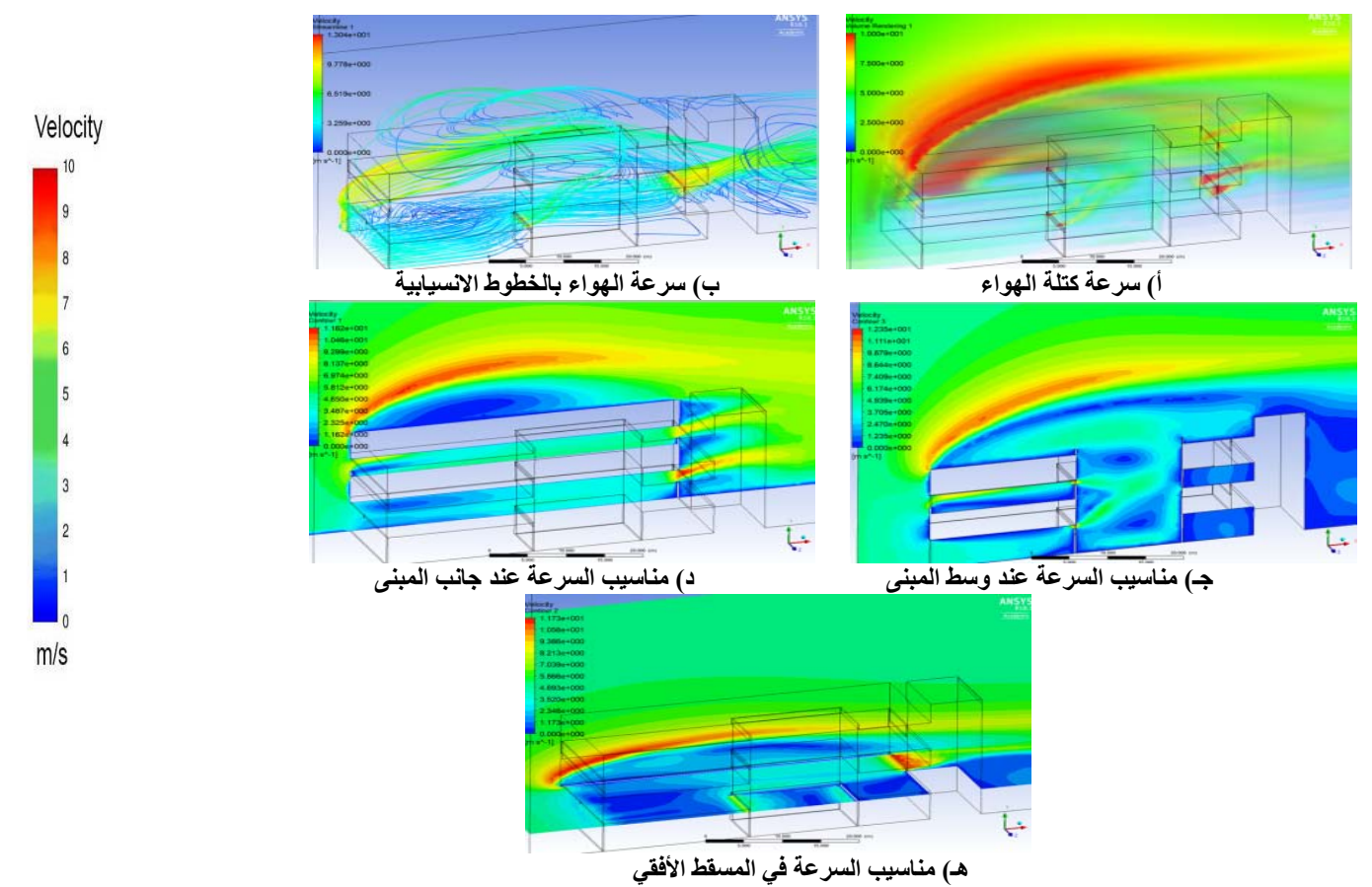

Ansys شكل (10) لقطات توضح مسار وسر عات الهواء في مقاطع مختلفة خارج وداخل المبنى للحالة الثانية, برنامج

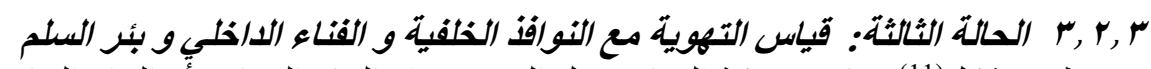

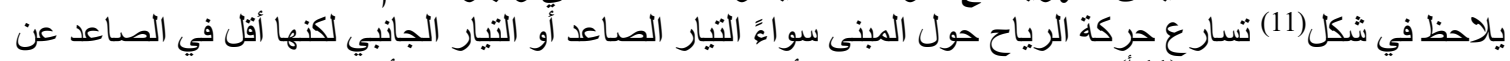

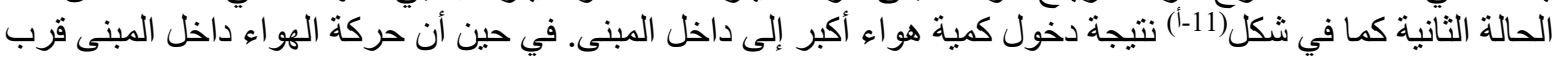

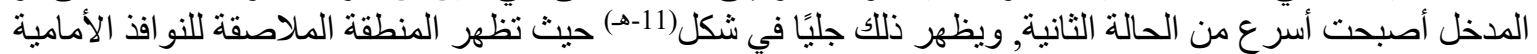

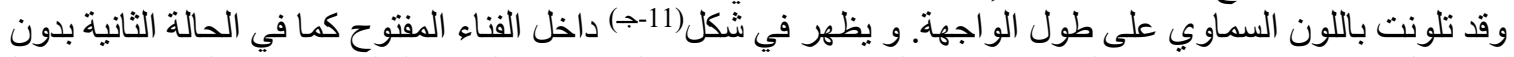

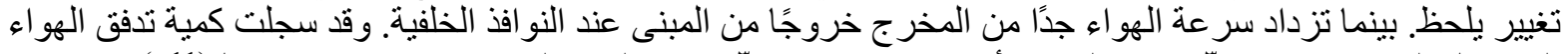

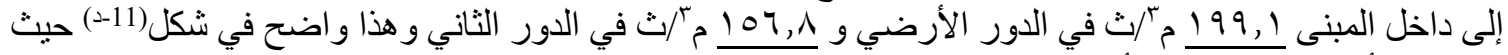
التحسن الأكبر حدث للأدور الأرضي.

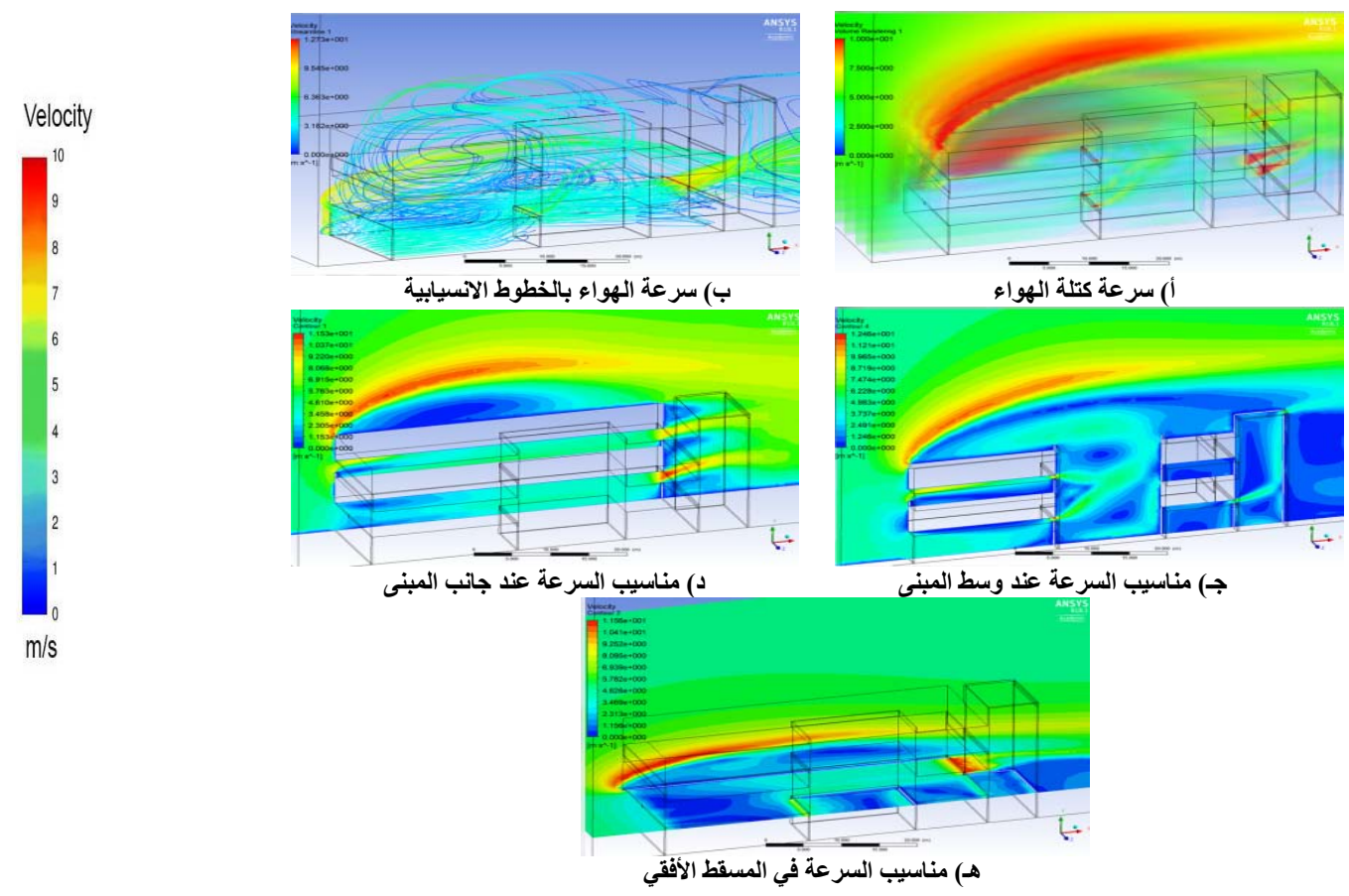

Ansys شكل (11) لقطات توضح مسار وسر عات الهواء في مقاطع مختلفة خارج وداخل المبنى للحالة الثانية, برنامج 


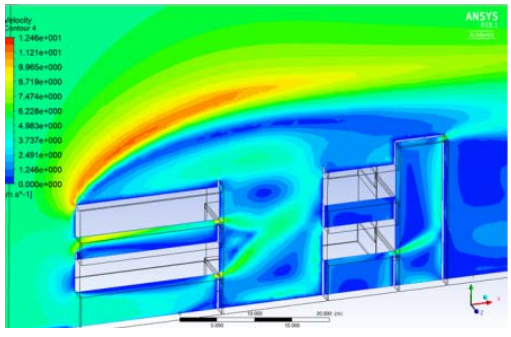

الحالة الثالثة: نافذة + منور + بئر سلم

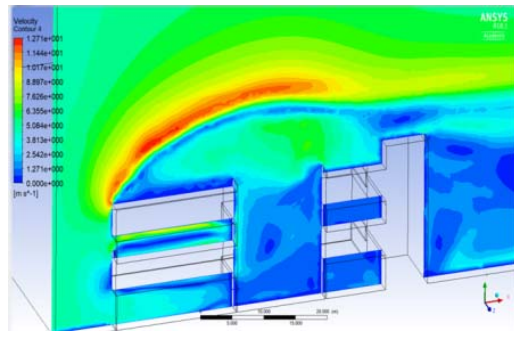

الحالة الثانية: نافذة + منور

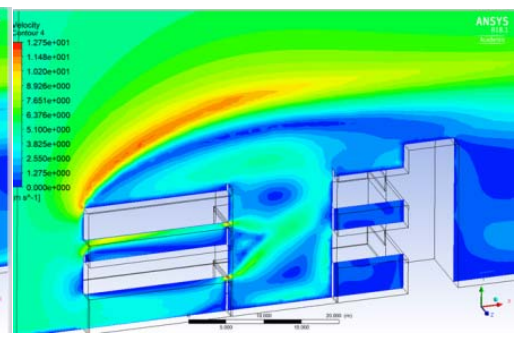

الحالة الأولى: نافذة

\section{Ansys شكل (12) مقارنة لسرعات الهواء خارج وداخل المبنى للحالات الثلاثة, برنامج}

\section{بمقارنة الحالات الثلاثة شكل(2) يلاحظ الآتي:}

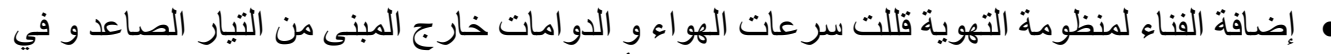

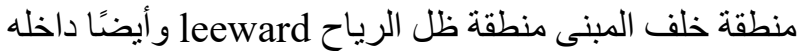

• ريادة كمية الهو اء المتدفق إلى داخل المبنى و زيادة سر عته نتيجة حدوث حركة للهو اء داخل الفناء من

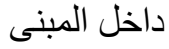

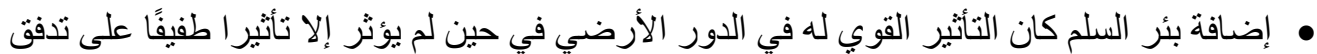

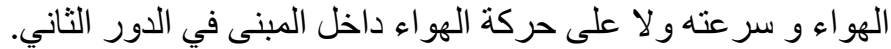

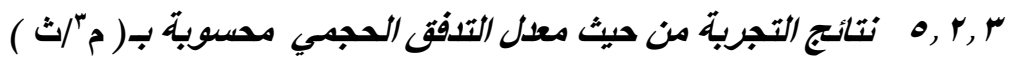

\section{Aدول ( ) مقارنة مخرجات معدل التدفق الحجسي للحالات الثلاثة في الأدوار العلوية والسفلية, برنامج Ansys}

\begin{tabular}{|c|c|c|}
\hline الدور الثاني & الدور الثاني & الحالات \\
\hline 115.6 & 140.6 & الحالة الأولي \\
\hline 153.2 & 171.1 & الحالة الثانية \\
\hline 156.8 & 199.1 & الحالة الثالثة \\
\hline
\end{tabular}

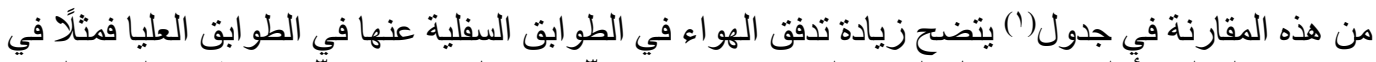

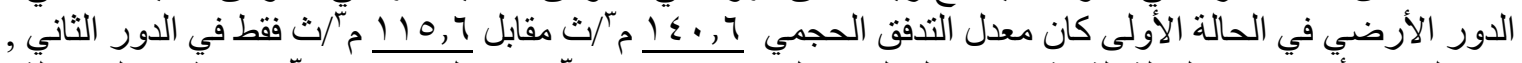

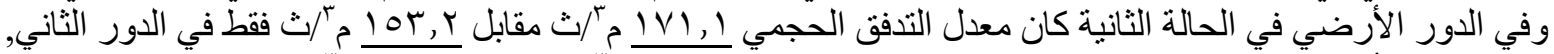

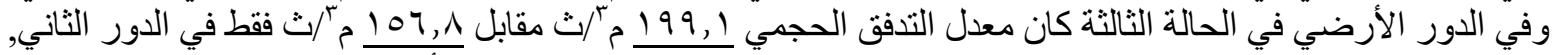
وسبب ذلك حدوث دو امات للهو اء خارج المبنى نتيجة اصطدامه به وارتفاع الهو اء إلى أعلى كتيار صاعد نظرًا لقصر

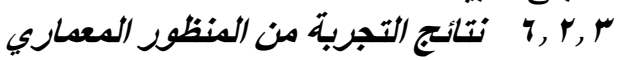

جدول (ץ) مقارنة للحالات الثلاثة في الأدوار العلوية والسفلية لزيادة التهوية الطبيعية مقابل زيادة نسبة الفتحات

\begin{tabular}{|c|c|c|c|}
\hline في الدور الثاني & في الدور الأرضية & 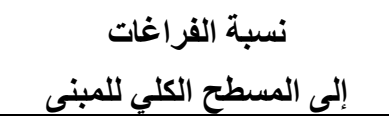 & الحالات \\
\hline زيادة ץr \% & زيادة r ز \% & زيادة من صفر إلى ع ا \% & لــ من الحالة الأولى إلى الثانية \\
\hline زيادة ץ \% & زيادة 17 \% & زيادة من ع ا \% إلى V V \% & ـــ من الحالة الثانية إلى الثالثة \\
\hline زيادة VT \% & زيادة r؟ \% & زيادة من صفر إلى V V \% & لــ من الحالة الأولى إلى الثالثة \\
\hline
\end{tabular}


لاحظنا في جدول(') أن التهوية الطبيعية للمبنى بدون فر اغات كما في الحالة الأولى زائدة في الطوابق السفلية

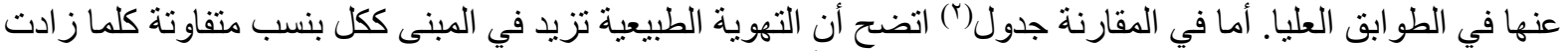

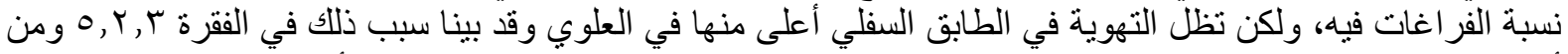

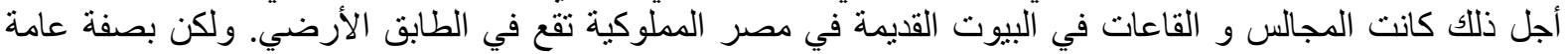

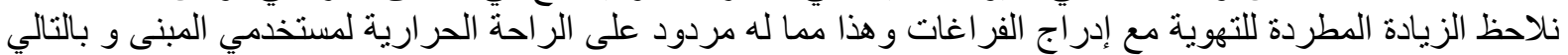
التوفير في الطاقة و الحفاظ على البيئة و الصحة الصنة العامة.

ا ـ هذه الدراسة حققت القياس الكمي و ليس الوصفي فقط لحركة الهو اء داخل المبنى مما أمكن معه عمل مقارنة

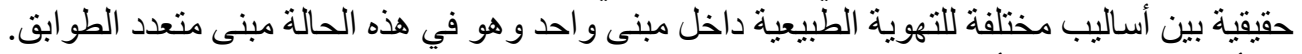

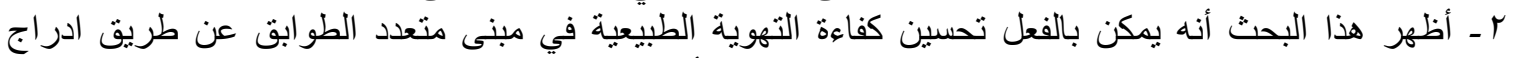

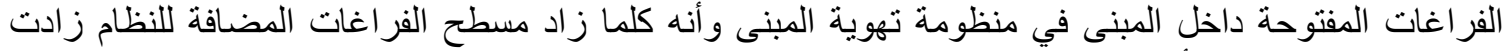

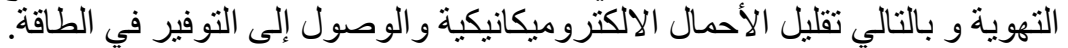

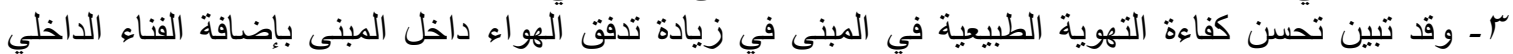

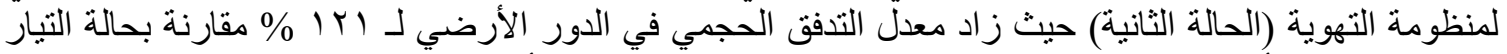

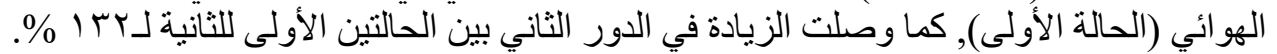

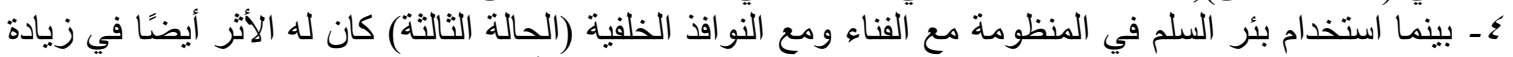

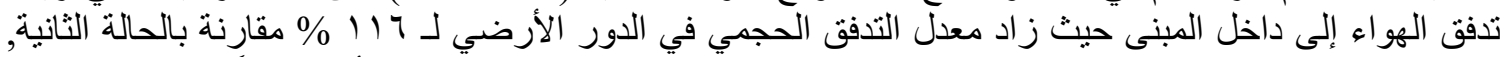

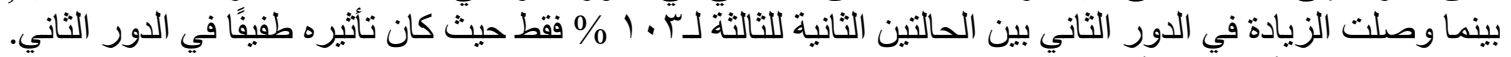

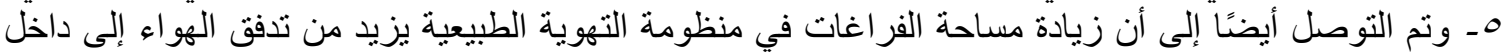

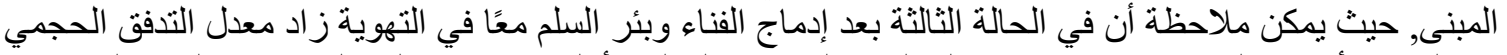

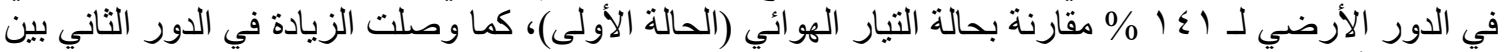

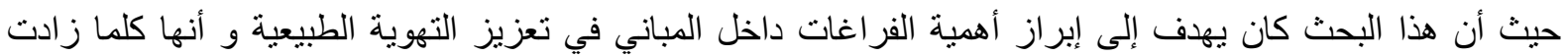

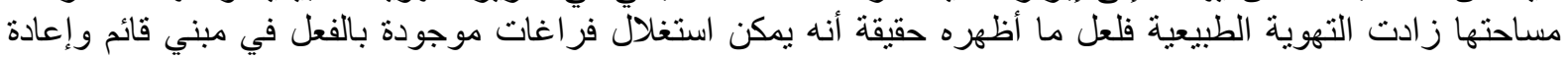

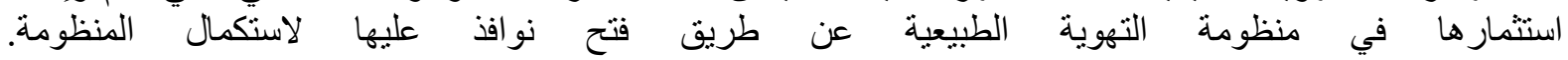

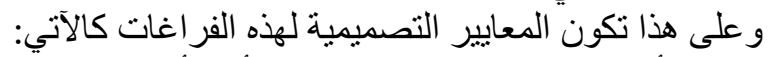

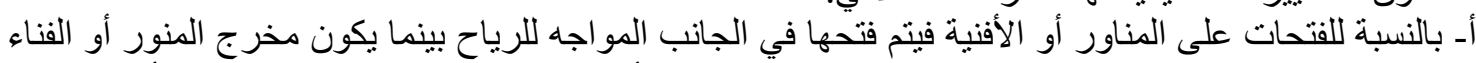

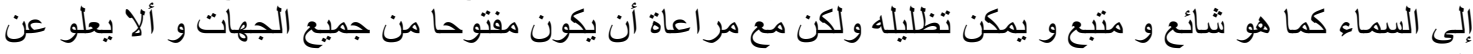
المبنى.

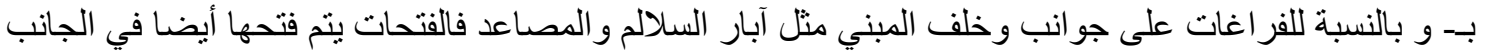

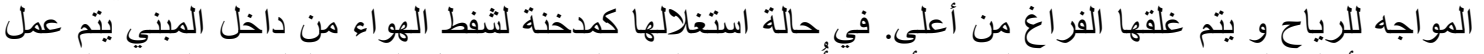

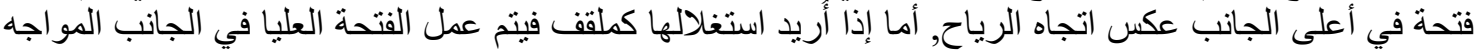
للرياح. وفي الحالتين يتم رفع جو انب هذا الفر اغ عن المبني بقدر طابق وكلما زاد الد كلما كان أفضل.

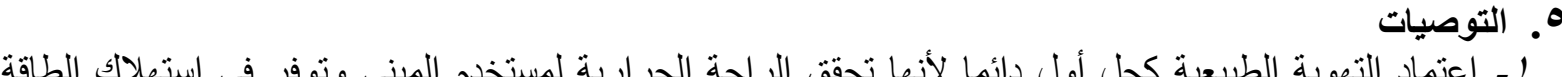
وتحافظ على البيئة. r - إدر اج الفراغات في منظومة التهوية الطبيعية لتعزيز التهوية وتحقيق الراحة الحرارية وتوفير التظليل لمستخدمي المباني. كــ إدراج أكثر من نوع مختلف من الفراغات لمنظومة التهوية الطبيعية للمبني يزيد من كفاءة تهوية المبنى.

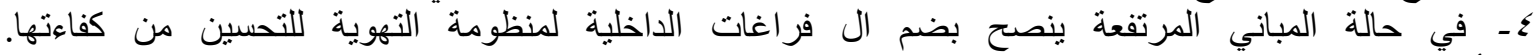

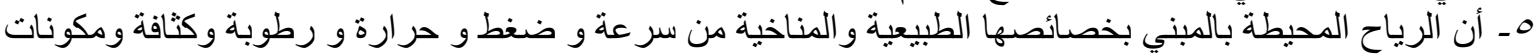

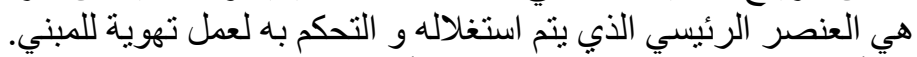

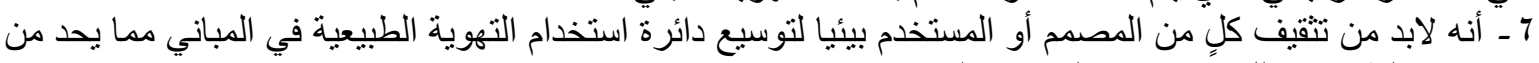

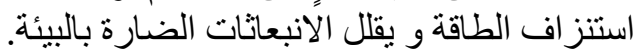




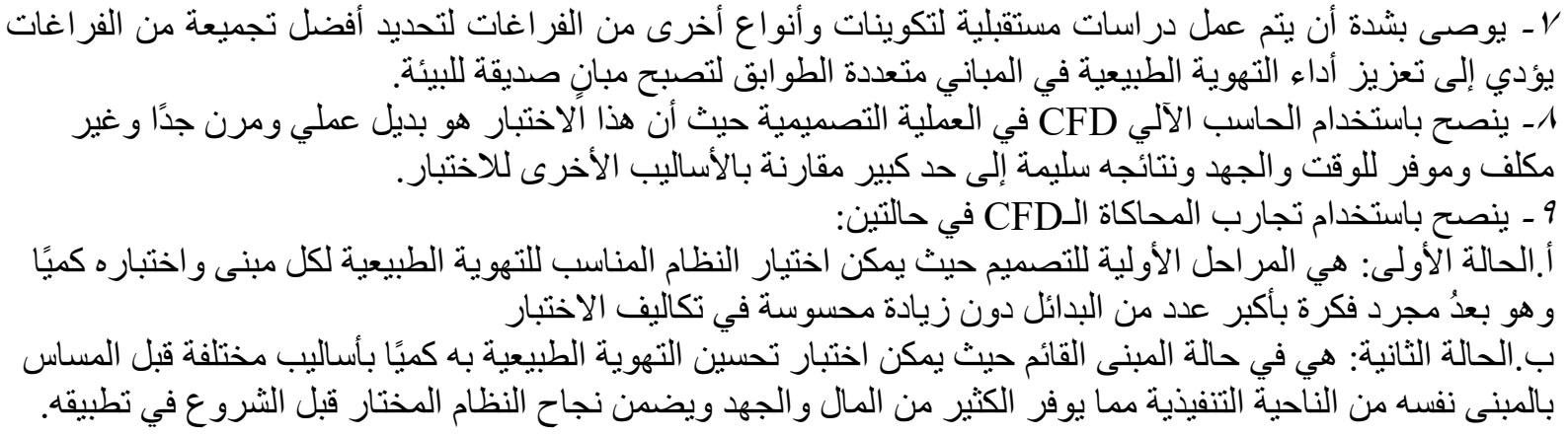

[1] Jamaludin, A. A., Hussein, H., Ariffin, A. R. M., \& Keumala, N. (2014). A study on different natural ventilation approaches at a residential college building with the internal courtyard arrangement. Energy and Buildings, 72, 340-352.

[2] Wang, H., Lin, H., Ng, V. C., Yang, T., \& Guan, L. Y. (2015). Failure of natural ventilation strategy in a sustainable house in China. International Journal of Low-Carbon Technologies, 10(3), 216-228.

[3] Butler, Kyle and Dagnew, Agerneh. march 22, 2013. Where the Wild Winds Blow, Part I. Air Worldwide. [Online]. https://www.air-worldwide.com/publications/aircurrents/2013/where-the-wild-winds-blow-part-i/.

[4] Macquoy, B. (2014). Natural ventilation based bioclimatic redevelopment: Building transformation and improvement into an integrated energy efficient multifunctional design, Delft University of Technology, Department of Architecture.

[5] Karakatsanis, C., Bahadori, M. N., \& Vickery, B. J. (1986). Evaluation of pressure coefficients and estimation of air flow rates in buildings employing wind towers. Solar Energy, 37(5), 363-374.

[6] Montazeri, H. (2011). Experimental and numerical study on natural ventilation performance of various multi-opening wind catchers. Building and Environment, 46(2), 370-378.

[7] van Hooff, T., Blocken, B., Aanen, L., \& Bronsema, B. (2011). A venturi-shaped roof for wind-induced natural ventilation of buildings: wind tunnel and CFD evaluation of different design configurations. Building and Environment, 46(9), 1797-1807.

[8] Perén, J. I., van Hooff, T., Leite, B. C. C., \& Blocken, B. (2016). CFD simulation of wind-driven upward cross ventilation and its enhancement in long buildings: Impact of single-span versus double-span leeward sawtooth roof and opening ratio. Building and Environment, 96, 142-156.

[9] Etheridge, D. (2011). Natural ventilation of buildings: theory, measurement and design. John Wiley \& Sons.

[10] Moosavi, L., Mahyuddin, N., Ab Ghafar, N., \& Ismail, M. A. (2014). Thermal performance of atria: An overview of natural ventilation effective designs. Renewable and Sustainable Energy Reviews, 34, 654-670.

[11] Ray, S. D., Gong, N. W., Glicksman, L. R., \& Paradiso, J. A. (2014). Experimental characterization of full-scale naturally ventilated atrium and validation of CFD simulations. Energy and Buildings, 69, 285-291.

[12] Ahmad, S., Badshah, S., \& Chohan, G. Y. (2014). Modeling and simulation of natural ventilation of building using solar chimney. World Applied Sciences Journal, 32(5), 741-746. 
[13] Ismail, A. M. (1996). Wind Driven Natural Ventilation in High-Rise Office Building With Special Reference To The Hot-Humid Climate of Malaysia, University of Wales College of Cardiff (Doctoral dissertation, $\mathrm{PhD}$, Thesis).

[14] Kotani, H., Satoh, R., \& Yamanaka, T. (2003). Natural ventilation of light well in highrise apartment building. Energy and Buildings, 35(4), 427-434.

[15] Murakami, S., Kato, S., Ooka, R., \& Shiraishi, Y. (2004). Design of a porous-type residential building model with low environmental load in hot and humid Asia. Energy and buildings, 36(12), 1181-1189.

[16] Hirano, T., Kato, S., Murakami, S., Ikaga, T., \& Shiraishi, Y. (2006). A study on a porous residential building model in hot and humid regions: Part 1 - the natural ventilation performance and the cooling load reduction effect of the building model. Building and Environment, 41(1), 21-32.

[17] Sadafi, N., Salleh, E., Haw, L. C., \& Jaafar, Z. (2011). Evaluating thermal effects of internal courtyard in a tropical terrace house by computational simulation. Energy and Buildings, 43(4), 887-893.

[18] Crasto, G. (2007). Numerical simulations of the atmospheric boundary layer. Universita degli Studi di Cagliari: Cagliari, Italy.

[19] Jatupatwarangkul, S., Arch, D., \& Tran, T. (2013). Project Deliverable No. 1: Literature Review of External CFD. University of Hawaii, School of Architecture.

[20] Shawon, M. J., El Chaar, L., \& Lamont, L. A. (2013). Overview of wind energy and its cost in the Middle East. Sustainable Energy Technologies and Assessments, 2, 1-11.

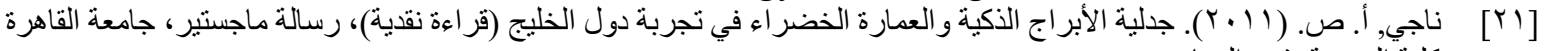

